\title{
Erhard Denninger Der Einzelne und das allgemeine Gesetz
}

$I$.

Vom Rechtsstaat ist hier dic Redc, von der Demokratic und von dem, was beide zu cinem frcihcitlichen Gemeinwesen zusammenfügt: von dem wallgemeincn ", "für allc geltenden « GESETZ.' Im Deutschland des Jahres 1995 scheint die große Rechrsunsicherheit ausgebrochen zu sein: Unsicher, ungewiß ist, so scheint es, dic Grenze geworden, die das Geserz zwischen strafbarem und nicht strafbarem Verhalten ziehen soll. Sitzblockaden vor cinem Munitionsdepot sind niche als Nötigung strafbar, ${ }^{2}$ abcr wic steht es mit den wütenden LKW-Fahrern, die eine Fernverkehrsstraße tagelang blockieren? Ein Auto-Aufkleber mit dem Tucholsky-Zitat "Soldaten sind Mörder « stcllt u. U. keine strafbare Beleidigung dar, sondern nur einen Ausdruck pazifistischer Grundgesinnung.' Unsıcher in ihrem Rechtsdurchsetzungswillen auch dieser gehört zur Grundausrüstung des Rechtsstaats! - zeigen sich Ausländerbehörden angesichss des von einigen hundert, meist evangelischen Pfarrern und Gemeinden in Anspruch genommenen und zugunsten von rechtskräftig ausreisepflichtigen Asylbewerbern praktizierten "Kirchenasyls". Unsicher und zwiespältig hinsichtlich der Lagebeurteilung und der zu ergrcifenden Maßnahmen reagiert dic Hannover'sche Polizci auf die von "Punkern " und »autonomen "Szene-Gruppen angckündigten und als solche inszenierten "Chaos-Tage«. Derlei Unsicherheit eincr Polizeipraxis ist allcin sicher kein Grund, den Chaos-Tagen im Rahmen unserer Fragestellung besondere Aufmerksamkeit zu schenken. Das Bild ändert sich, wenn man feststellen muß, daß das Fehlverhalten der Polizei nichts anderes ist als der Reflex fundamental unterschiedlicher Finschätzungen verantworelicher Politiker. Während die einen dic Straßenbarrikaden, Brandsätze und Pflastersteine schlicht als "Straßenterror « behandelt und mit allen polizeilichen und strafrechtlichen Mitteln bekämpft wissen wollen, versuchen andere Landtagsabgeordnete, ein solches Jugend-Treffen mit bundesweitem Echo, über den Horizont der "öffentlichen Sichcrhcit « hinaus, als ein *kulturelles und Milieu-Ereignis* zu begreifen. ${ }^{4}$ Was für dic einen Anlaß ist, nach bayerischem oder säclisischem Vorbild die Ausdehnung des polizeilichen "Unterbindungsgewabrsams"s' (also das vorbcugende Einsperren eines, der möglicherweise

1 Vgl. Art. 5 Abs. 2 GG einersets, Art. 137 Abs. 3 Satz, 1 WRV in Verbindung nmt Art. 140 GG andererseits. Vgl. auch W. Bock, Das fur alle geltende Gesetz und die kırchliche Selbstbestımmung, Tubingen 1995. 2 BVerfG, Beschluß vom 10.1. 1995, NJW $1995,1141 \mathrm{ff}$.

3 BVerfG, Beschluß der 3 Kammer des Ersten Senats vom 25. 8. 1994, Az : 1 BvR $1423 / 92$.

4 Vgl. MdL Jordan It. Beracht der Frankfurter Algemeinen Zeitung vom 8. 8. 1995, S. 1.

5 Vgl. Art. 17, 20 Bayerisches Polizciaufgabengesetz vom 14 9. 1990; $\$ 22$ Sachsisches Polrzcigesetz vom 15.8. 1994 ; kriusch dazu E. Dennunger, Normbesummthell und Verhaltnismaßigkeitsgrundsatz im Sachsischen Polizergesetz?, Leipziger Juristische Vortrage Nr. 10, 1995: ferner: M. Walzer, Spharen der Gerechugkett, 1992, S. 386 f., 387: "Die Gefangensetzung ist in diesem Fall nichts anderes als ein Akt der Tyrannes.a 
sonst zum Straftäter werden könnte!) auf die Dauer von z.wei Wochen zu fordern, ist für die anderen Anlaß, in Zusammenarbeit mit Sozialarbeitern ein Konzept für künftige "Chaos-Tagex auszuarbeiten, nach welchem den als an sich friedlich eingeschätzten Punkern "öffentlicher Raum in der Stadt gewährt werden solle, wo sie sich ungestört sollen entfalten können. Unsicherheit im politischen und rechtlichen Umgang mit Minderheiten, alten wie sneuen $*^{6}$, überall.

Ihren vorläufigen Höhepunkt erreicht diese allgemeine Verunsicherung mit dem Bcschluß des Bundesverfassungsgerichts vom r6. Mai 1995, ' welcher Kreuze und Kruzifixe aus den Klassenzimmern bayerischer Volksschulen verbannt. Der Vorgang mit allen dazu bekannt gewordencn Reaktionen wiegt aus mehreren Gründen schwer: I. unter dem Aspekt der Gewaltenteilung. Er demonstriert, was nicht neu ist, die Macht des höchsten Gerichts, das sogar den Gesetzgeber »in scine Schranken « zu verweisen vermag. Aber hier licgt zugleich das Problem. Wer bestimmt diese Schranken? Die Verfassung, antwortet das Verfassungsgcricht, dic acht Richterinnen und Richter eines Senats, erwidern die Parlamentarier, oder, kritischer noch: die fünf Richter der Senatsmehrhcit, dic in einem von allerlei Proporzrücksichten geprägt, parteipolitisch austarierten Do-ut-des-Vcrfahren in ihr Amt gekonmen sind. Die Fülle ihrer Macht charakterisiert zugleich deren Grenzen. Sie werden durch dic Funktion des Gerichts als einer letzten, endgültigen Streitbefriedungs- und damit Rechtssicherheitsinstanz bcstimmt: Verfehlt das Gericht diese seine Aufgabe der endgültig-gültigen Streitbefriedung, so wird seine Entscheidung nicht mehr als Rechtsentscheidung, dic Rechtssicherheit schafft, akzeptiert, sondern nur noch als bloßer "Machtspruch" kommentiert. So ist seine Macht insowcit schon dahin. Dies zeigen, und das ist in dieser Schärfe neu, der Kruzifix-Beschluß und zahlreiche PolitikerKommentare dazu.

An dieser Stelle erscheint, gewissermaßen in Parenthese, eine Anmerkung zur Publizitätsfunktion des höchsten Gerichts am Platze. Gcrade wegen seiner Rolle als letztentscheidende "Schiedsinstanz" in einem pluralistischen Gemeinwesen wäre es verderblich, wenn das Gericht seine Praxis an der Maxime des „Fiat iustitia pereat mundus!« orientieren wollte. ${ }^{8}$ Dies darf andererseits nicht als Ermuntcrung verstanden werden, verfassungsrechtliche Konflikte in politische bargaining-Prozesse aufzulösen. Der »informale Rechesstaat " hat im Karlsruher Glaspalast mit seiner symbolischen Transparenz nichts zu suchen. Wohl aber ist daran zu erinnern, daß das Gericht die Reaktionen einer mit den Einzelheiten des Falles nicht vertrauten Öffentlichkeit, die gewissermaßen nur die spektakuläre Schlagzeile aufnehmen kann und will, besser einzuschätzen und seinerseits antizipativ informierend darauf cinzugehen lernt. Das Gericht muß sich aktiver als bisher darauf einstellen, daß scine Entscheidungen unmittelbare Wirkungen im politischen Raum entfalten, und das heißt auch: daß sic kritisiert oder als politische Waffe benut/l werden, bevor sie überhaupt (sorgfältig) gelesen worden sind. Oft wird ein einzelncs normatives Element der Entscheidung aus seinem Zusammenhang gurissen, vergröbert und unzulässig verallgemeinert. So hat das Gericht z.B. niemals verkündet, man dürfe Soldaten jederzeit ungestraft als Mörder bezeichnen, sondern es hat dem inkriminierten Zitat in einem individuell bestimmten Kontext eine mögliche fundamental-

6 Der Terminus „Neue Minderhetten wird gebraucht von H.Schulze-Fielitz, in: Th. Fleiner-Gerster (Hrsg.), Die multikulturelle und multethnische Gesellschaft, PIFF, Fribourg 1995, S. 139.

7 1 BvR 1087/91, EuGRZ 1995, 359. Im gleıchen Sinne wie jetzt das BVerfG hatte das Schweizerische Bundesgericht bereits am 26.9.1990 entschieden, vgl. EuGRZ 1991, 89 ff.

8 D. Grumm, Entscheidungsfolgen als Rechtsgrunde: Zur Argumentationspraxis des deutschen Bundesverfassungsgerichts, in: G. Teubner (Hrsg.), Entscheidungsfolgen als Rechtsgrunde, 1995, S. 139 ff., weist die Folgenberucksichugung als seit langem gefestigten Bestandteil der Grundrechtsjudikatur des BVerfG nach. 
pazifistische Interpretation gegeben und das für die Sache zuständige Strafgericht dazu angehalten, den Grundsatz "In dubio pro reo" zu beachten. Es geht nicht darum, daß das Bundesverfassungsgericht wegen der angeblichen Mchrdeutigkeit seiner Urtcilc eine Art authentischer Interpretation derselben geben sollte, sondern zu fordern ist eine um äußerste Klarheit bemühte, für jeden nicht »böswilligen « Bürger nachvollzichbare Präsentation der Argumente, welche der Institution die lebensnotwendige Akzeptanz sichert. So sollten die Sondervoten nicht als esoterische Fortsetzung der senatsinternen Diskussion für die Richterkollegen geschrieben werden, sondern zur Verdeutlichung der unübcrbrückbaren Dissenspunkte für jedermann. ${ }^{9}$ Ohnehin bleibt es dann Aufgabe der Wissenschaft, sowohl die dogmatische Feinkommentierung als auch die Einbettung der punktuellen Verfassungsinterpretation durch das Gericht in den gesamten Kontext der rechtlichen und gesellschaftswissenschaftichen Theoriediskussion zu leisten. Zu dem letzteren soll das Folgende beitragen.

2. Der zweite Gesichtspunkt, der dem Kruzifix-Beschluß und seiner Rezeption exemplarische Bedeutung verleiht, geht weit über das immer spannungsvolle Verhältnis Verfassungsgerichtsbarkeit - Gesetzgeber hinaus. Man erfaßt ihn, wenn man das Gericht nicht als Schiedsinstanz, als "Austrägalinstanz, * ${ }^{\text {ro }}$ sondern emphatisch als "Hürer der Grundrechte, besonders der Minderheit «, begreift" und demgegenüber den Gesetzgeber als Instrument des politischen Mehrheitswillens, der mit dem Anspruch auftritt, Ausdruck der "volonté générale « zu sein. Vernachlässigt man einmal, daß die vom Bundesverfassungsgericht für nichtig erklärtc Norm ( $\mathbb{1}$ r3 Abs I, S. 3) der Bayerischen Volksschulordnung vom 2 1. 6. I983 nicht selbst vom Parlament erlassen, sondern im Wege der Rechtsverordnung ergangen war, dann kann man einen großen Teil der Urteilskritik als Ausdruck des Erschreckens, ja des Entsetzens der (bisher meist schweigenden) Mehrheit darüber begreifen, daß sie einer Minderheit, hier durch ein einziges Elternpaar mit schulpflichtigen Kindern verkörpert, weichen soll. Wird hier nicht das Grundprinzip demokratischer Entscheidungsfindung pervertiert? Bereitet hier nicht cin "Urteil der Intoleranz", wie es der Bayerische Ministerpräsident Stoiber genannt hat, ${ }^{2}$ der "Intolcranz einer kleinen Minderheit " den Boden gegenüber einer ganz großen Mchrheit, dic ihren Willen nicht nur gesetzlich, sondern sogar in der Landesverfassung deutlich kundgetan hatte? Art. 135 BayVerf sieht die Unterrichtung und Erzichung der Schüler »nach den Grundsätzen der christlichen Bekenntnisse vor «. Wiegt dieser Verstoß, so die Kritiker, gegen das demokratische Mehrheitsprinzip nicht um so schwerer, als es hicr, mit Hilfe des Gerichts, einer "winzigen Minderheit", einer einzigen Familie, gelungen ist, der überwältigenden Mehrhcit der Bevölkerung des Landes eine Verhaltensweise aufzuzwingen, die ihren grundlegenden Überzeugungen widerspricht? Liegt dieser Fall der Durchsetzung "ncgativer "Glaubensfreiheit weniger Einzelner deshalb nicht wesentlich anders als der Streit um die optimale Lebensschutzregelung durch einc Abtreibungsgesetzgebung, die 1992 mit beträchtlicher parteienübergreifender Mehrheit' ${ }^{3}$ beschlossen, dann jedoch von einer ebenfalls beträchtlichen parlamentarischen Minderheit vor dem Verfassungsgericht erfolgreich angefochten worden war?

9 Als Beispiel fur die hıer kritısıerte Praxıs kann das abweichende Votum der Rıchterın Graßhof zu der Cannabis-Entscheidung des BVerfG angesehen werden: BVerfGE 90, $145,199 \mathrm{ff}$.

10 Vgl. etwa Art. XI der Deutschen Bundesakte vom 8.6.18 is und die dazu ergangene Austragal-Ordnung vom 16.6 .1817 ,

11 In diesem Sinne jungst auch G. Frankenberg, Lowen unter dem Thron, Frankfurter Rundschau vom 20. 9. 1995 , Nr. 219 : Minderheitenschutz ist der vornehmste und unbequeme Beruf von Verfassungsrichterinnen und -richtern.s

$12 \mathrm{Vgl}$. DER SPIEGEL Nr. $33 / 1995, \mathrm{~S} .33 \mathrm{f}$.

13 Das Schwangeren- und Famulienhilfegesetz vom 27. Juh 1992 wurde in namentlicher Abstımmung mit 357 Ja-Stimmen gegen 284 Nem-Stummen bei 16 Enthaltungen beschlossen. Vgl. BVerfGE 88,203 , 223. 
Diesc und ähnliche Einwände offenbaren ein elementares Unverständnis hinsichtlich des Verhältnisses zwischen Rechtsstaat und Demokratic, zwischen dem individuellen Menschenrechts- und Grundrechtsstatus des Einzelnen und der Funktion des nach Mehrheitsregeln zustandegekommenen allgemeinen Gcsetzes:

a) Die Gewährleistung des unabdingbarcn Grundrechtsschur/es - das Grundgesetz spricht vorn "Wesensgehalt e eines Grundrechtes, Art. 9 Abs. 2 - ist keine Frage der Quantität (der Schutzsuchenden). Die Verletzung in einem einzigen Fall wiegt, normativ gesehen, genauso schwer wie massenhafte Verstöße. Dieser Kernbereich eines Grundrechts ist einer Entscheidung nach Majoritätsregeln genauso unzugänglich wie Fragen der wissenschaftlichen Wahrheit, über die auch nicht abgestimmt werden kann." Das Bundesverfassungsgericht hat in bciden Urteilen zur Schwangerschaftsunterbrechung's diesen Gedanken der Nichtquantifizierbarkeit in den Mittelpunkt seiner Argumentation gerückt, indem es fordert, das Leben das Ungeborenen müsse in jedem konkreten Einzelfall und nicht nur statistisch geschützt werden. Entsprechendes gilt für die Religions- und Glaubensfreiheit in ihrem Kernbereich: Über Glaubens- und Gewissensfragen, also über »den rechten Glauben « und scine Inhalte, kann es keinen Mehrheitsentscheid geben. Greift eine staatliche Maßnahme, und sei es aufgrund cines Gesetzes, in den absolut geschützten Kernbereich der Rechte auch nur eines einzigen Bürgers ein, so muß sie insowen weichen. Insoweit kommt die vom Grundgesetz - im Vergleich zur Weimarer Reichsverfassung - vurstärkte Geltungskraft der Grundrechre zum Tragen, die gemäß Art. I Abs. 3 GG nicht nur Verwaltung und Rechtsprechung, sondern auch den Gesetzgeber als »unmittelbar geltendes Recht « binden. Eine zweite, ganz andere Frage ist, auf welche Wcisc der Grundrechtsverletzung abzuhelfen ist.

b) Jenseits der absolut zu schüt\%enden Kernzone eines Grundrcchts öffnet sich ein breites Spektrum möglicher Konflikte zwischen verschicdenen Grundrechten und Grundrechtsträgern, aber auch zwischen Grundrechten einerseits und verfassungsrechtlich (nur objcktivrechtlich) geschützten Gemcinwohlgütern andererseits. In allen Fällen ist es in erster Linie Sache des Gesetzgebers, für einen Ausgleich der widerstreitenden Werte und Interessen nach dem Grundsatz der »praktischen Konkordanz (K. Hesse) $)^{16}$ zu sorgen. Hinsichtlich des Ausgleichs in Religions- und Weltanschauungskonflikten gilt das an alle Bürger gerichtetc Tolcranzgebot und das an den Staat adressierte Neutralitätsgebot. Dies wird auch übereinstimmend und nahezu wortgleich sowohl von der Mehrheit wie von der überstimmten Minderheit ( $s: 3)$ der Richter des umstrittcnen Kruzifix-Beschlusses so festgestellt. ${ }^{17}$ Wie ist es dann aber möglich, daß beide Teile dennoch zu diametral entgegengesetzten Ergebnissen gelangen?

Die Differenz, in der Interpretation und Anwendung des Toleranzgebotes bezeichnet genau den Punkt, welcher dem Beschluß das größte sowohl grundrechts- wie demokratietheoretische Interesse verschafft. Man übertreibt nicht mit der Feststellung, daß die Divergenz im Ersten Senat des Bundesverfassungsgerichts, ausbuchstabiert an der Spannung zwischen positiver und negativer Glaubensfreiheit, zwei grundsätzlich verschiedene Demokraticverständnisse sichtbar werden läßt, welche man in der Terminologie der aktuellen Theoriediskussion als "liberal" bzw. als "kommunitaristisch ; bezeichnen kann. Natürlich findet sich in der Entscheidung darüber kein

14 Vgl. BVerfGE 35, 79, 148ff., 151, abweichende Meinung des Richters H. Simon und der Rıchterın W. Rupp-v. Brunneck zum Hochschulgesetz-Urtel.

is BVerfGE 39, 1, $88 \mathrm{ff}, ; 88,203$, LS 2, 252.

$16 \mathrm{~K}$. Hesse, Grundzuge des Verfassungsrechts der Bundesrepublık Deutschland, 20 Aufl., 1995, Rdnr. 317 ff.

17 Vgl. BVerfG, EuGRZ 2995,365 re. Sp. and 367. 
Wort, und einige der Richterinnen oder Richter mögen sich durch meine nunmehr "metajuristisch « ansetzende Kritik übcrinterpretiert oder einfach ganz mißverstanden fühlen. Darauf kommt es hier nicht an; entschcidend ist, was die verlautbarten Texte der Mehrheits- und der abweichenden Minderheitsargumentacion hergeben.

Dic Minderheitsmeinung, die wir hicr einmal für den kommunitaristischen Standpunkt in Anspruch nehmen dürfen, geht von einer das ganze Gemeinwesen beherrschenden homogenen religiösen Grundüberzeugung aus, auf die auch dic Landesverfassung Bezug nimmt, indem sie Unterrichtung und Erriehung der Kinder nach den Grundsätzen der christlichen Bekenntnisse vorschreibt. (Inwicwcit dicse Annahme auch nur für Bayern faktisch zutrifft, spielt keine Rolle.)

Auf der Basis gleichartiger Überzeugungen einer großen, unangefochtenen Mchrheit läßt sich eine stabile Rechtsordnung aufbauen. ${ }^{18}$ Das "allgemeine Gesetz« ist das, von wenigen Ausnahmen abgesehen, allgemein akzeptierte Gesetz. Die richterlichen Urteile, die sich in scinem Rahmen bewegen, dürfen (fast) allgemeiner Zustimmung sicher sein. Auch in ein solches Rechtssystem läßt sich ein Toleranzprogramm einbauen, jedoch in folgender Weise: Die Mehrheit stolericrt die Minderheit(en), indem sie deren Existenz duldet und ihnen ein Minimum an Rechten zuerkennt. Keineswegs bedcutet diese Duldung durch dic Mehrheit, daß den Minderheiten die volle Gleichberechtigung eingeräumt wird. Die Minderheit wird als Ausnahme behandelt, die z.war sprichwörtlich die Rcgel bestätigt, rechtlich aber in der Weise, daß die "Regel ", also der Mehrheitskonsens, um so unverbrüchlicher durchgesetzt wird. "Toleranz «edeutet auf dieser - ich würdc sagen: - Entwicklungsstufe des demokratischen Bewußtseins, daß die geduldete Minderheit es hinzunehmen hat, daß die Mehrheit sich voll, auch mit Unterstützung des Staates, entfalten kann, während sie selbst ein zwar rechtlich gesichertes, aber doch ein "Außenseiterdasein " fristen muß. Charakteristisch und folgenreich ist die Behandlung des abweichenden Minderheitsverhaltens als "Ausnahmeerscheinung", mit der dic Rechtsordnung »irgendwie" fertig werden muß. Dadurch wird es von vornherein als das "Abnorme «, als von der Norm Abweichende, ja die Norm Negierende stigmatisiert und, wo nicht sogleich ins Unrecht gesetzt (wie bei den nach ordnungsgemäßer Beratung den Schwangerschaftsabbruch verlangenden Frauen'9), so doch in die Grau- und Randzone rechtlicher Minderwertigkeit abgedrängt.

Unter diesem Aspekt von Regel und Ausnahme wäre die Geschichtc von Minderheiten in der Bundesrepublik in den letzten 40 Jahren neu zu schreiben, die Geschichte der Kricysdienstverweigerer beispielsweise, trotz und wegen der Freistellungsgarantic des Art. 4 Abs. 3 GG. Oder die Geschichtc der Homosexuellen oder der Transsexuellen und anderer Gruppen. Die Geschichte der Kriegsdienstverweigerer oder, "positiv" gesagt: der Zivildienstleistenden - freilich gcradc nicht die Geschichte der "Totalverweigerer " (also der auch den Ersatzdienst verweigernden Pazifisten) - zcigt aber auch, daß eine Gesellschaft und ihr Recht zu allmählichen kollektiven Lernprozessen in der Lage sind. Zum einen mußtc allein schon das Anschwellen der Verweigererzahlen in den yoer Jahren auf etliche Zehntausende jährlich ${ }^{20}$ die Tiefe der Kluft zwischen »Normalität « und »Abnornität « immer fragwürdiger werden lassen; zum anderen veränderte die zehntausendfach bewiesene

18 Unter Hinweıs auf Rousseau (s. u.) forderı Carl Schmit fur dıe Stabilıtat der Demokratie eın moghichst hohes Maß an ssubstanzielle(r) Gleichartigkeit des Volkes*, vgl. Verfassungslehre, 1928, S. 214 f.

19 BVerfGE 88, 203, 273, 279: Die Folge der Rechtswidrigkeit bekommt die Frau nicht als Strafe, wohl aber sin den ubrigen Bereichen der Rechtsordnung= zu spuren.

20 Nach zeitweilıgem Absınken sınd die Verweigererzahlen jetzt wieder im Ansteıgen begriffen: 1994 wurden ca. 125000 Verweigerer festgestellt, im 1. Halbjahr t995 bereits uber 85000 , vgl. Das Parlament, 45. Ig. Nr. $36 / 37$, S. T. 
gesellschaftliche Nützlichkeit, ja zum Teil Unersetzbarkeit der Dienstleistungen der Verweigerer im Alten- und Krankenpflegesystem des überforderten Sozialstaats die Akzeptanz und das Verständnis für die Kriegsdienstverweigerer mindestens (aber nicht nur) bei den Nutznießern der Dienstleistungen. Freilich, so weit sind wir noch nicht, daß der Zivildienst den gleichen Karrierefördereffekt hätte, wic, zu Kaiser Wilhelms Zeiten, auf die Standardfrage: "Wo haben Sie gedient?" die Antwort: "Im I. Gardekürassierregiment!"

Auf der Gegenseite darf man nicht übersehen, daß erst die Geltendmachung der "Ausnahme" die Vertcidiger der "Regel «, also die Mehrheit dazu veranlaßt, die Regel sowohl genauer zu definieren als auch zu "begründen «. Erst die massenhafte Kriegsdienstverweigerung und die daraus folgende gerichtliche Auseinandersctzung haben die demokratische Ideologie der allgemeinen Wehrpflicht und, unter dem Gedanken der Lastengerechtigkeit, auch des Ersatzdienstes hervorgebracht. ${ }^{21}$ Auch insofern bestätigt die Ausnahme die Regel, als jene diese bckräftigt, indem sic sic zur Begründung provoziert. Die hier als "kommunitaristisch « bezeichnete Denkweise ist also im allgemeinen wenig geneigt, einen Ausgleich der konträren Standpunkte auf der Linie gegenseitigen Nachgebens zu suchen, die Tolcranz auch durch die Rücknahme eigener vorgeschobener Positionen auf einen gemeinsamen Nenner mit der Minderheit zu üben. Im Beispiel des Kruzifix-Beschlusses »löst die Senatsminderhcit das Problem des Konflikts zwischen positiver und negativer Religionsfreiheit dementsprechend auch nicht dadurch, daß sie den staatlichen Raum, hier das Klassenzimmer, "religiös neutralisiert" und dadurch die Verletzung der religiösen Gefühle nichtchristlicher Schüler aus der Welt schaffr, sondern indem sic aus zwei zwar teilweise zustimmungswürdigen, aber inhaltsarmen "Grundsätzen" in theologisch, erzichungspsychologisch und norminterpretatorisch fragwürdiger Weise folgert, die Schüler der nichtchristlichen Minderheit seien zur Duldung des Kreuzes in) Klassenzimmer verpflichtet. ${ }^{22}$ Die beiden Grundsätze lauten: I. Die negative Religionsfreiheit ist kein „Obergrundrecht", das die positiven Äußerungen der Religionsfreiheit verdrängt. Abstrakt gesehen ist dies sicher richtig, die Richter berücksichtigen aber nicht, daß es hier um die Unterlassung eines grundrechtsverletzenden Eingriffs seitens des Staates geht. 2. Der zweite Grundsatz: "Das Recht der Religionsfreiheit ist kein Recht zur Verhinderung von Religion« ist, abstrakt gesehen, ebenfalls richtig. Aber hier fehlt der Zusatz, daß das Grundrecht der Religionsfreiheit auch nicht das Rechr gibr, die eigene Religion jederzeit und an beliebigem Ort wauf Kosten «, d. h. unter Verletzung der Religionsfreihcit anderer auszuüben. Theologisch fragwürdig ist die Minderheitsmeinung im Senat, weil sie die Bedeutung des Kreuzes in der Schule zu minimieren versucht, nämlich bloß als ein Sinnbild „für die Vermittlung der Worte der christlich geprägten abendländischen Kultur« in der Schule. Psychologisch und interpretationstheoretisch fragwürdig ist der anmaßende Versuch der Richter, die Symbolbedeutung für die nichtchristlichen Schüler autoritativ und damit das "Gewicht « ihrer "psychischen Bceinträchtigung und mentalen Belastung " zu bestimmen. Kreuze, so heißt es, hingen in Baycrn ohnehin überall, in Krankenhäusern wie in Gaststätten, Privathäusern und an öffentlichen Wegen. Die Kreuze in der Schule blieben mithin »im Rahmen des Üblichen «. Das Problem ist aber gerade, ob das "Übliche« in der Schule ohne weiteres den Rahmen bestimmen darf. Die Argumentation der Richterminderhcit ist typisch für die einer im übrigen überwältigenden Mehrheit gegenüber einer winzigen, deshalb anscheinend nicht ins Gewicht fallenden Minderheit. Doch die Sprache ist manchmal klüger als ihre Sprecher. Die

21 Vgl. BVerfGE 69, 1, 22;48, 127, 16, f.

22 BVerfG EuGRZ 1995, 359, Abw. Meinung 368. 
Rede von der rüberwältigenden Mehrheit soll nicht nur eine quantitative Größe bezeichnen; sie enthält auch eincn argumentativen Appell, sie soll von der Richtigkeit des Mehrheirsstandpunktes überzeugen. Die Sprache verrät zugleich auch die "Überwälrigung e der Minderhcit durch die Mehrheit; sie gibt Kunde von der Gewalt, mit der die Mehrheit sich durchsetzen will. Der "Rahmen des Üblichen « ist sozusagen das Sprachrohr der »schwcigenden Mehrheit«, die zugleich die überwältigende ist; sie schweigt und läßt „das Übliche " für sie sprechen.

Die Richtermehrheit, deren Votum dic Entscheidung trägt, nimmt demgegenüber den Charakter des Grundrechts als Minderheitenschutzrecht ernst. Der Staat, der die Glaubensfreiheit umfassend gewährleistet, verpflichtet sich damit selbst zu religiösweltanschaulicher Neutralität. ${ }^{23}$ Das Grundgesetz selbst hat diesen Gedanken jedoch nur unvollkommen umgesetzt, wenn es z. B. den (christlichen) Religionsunterricht als ordentliches Lehrfach vorschreibt (Art. 7 Abs. 3 Satz I) und die Einrichtung privater Ersatz-Volksschulen als Bekenntnis- oder Weltanschauungsschulen zuläßt (Art. 7 Abs. 5). Den Landesgesetzgebern ist die Einführung weiterer religiöser Elemente wie das morgendliche (freiwillige) Schulgebet unbenommen. Die Richter der Senatsmchrheit crkennen dies alles an, versuchen aber, den liberalen Grundansatz wieder deutlich zu machen. Staatliche Pflichtschule darf im religiös-weltanschaulichen Bereich ilure Aufgabe nicht »missionarisch auffassen « und »keine Verbindlichkeit für christlichc Glaubensinhalte beanspruchen «. Nur das »unerläßliche Minimum an $Z_{\text {wangselementen }}{ }^{24}$ dürfe den Kindern, die selbst oder deren Eltern keine christliche Erziehung wünschen, zugemutet werden. Der Zwang, die eigene abweichende Religionsauffassung offenbaren zu nuüssen, inden das Kind dem Schulgebet der Mitschülcr fernbleibr, halte sich in diesem Rahmen"s, der Zwang hingegen, wunter dem Kreuz lernen zu müssen«, der nicht einmal cine Offenlegung der "Andersheit« erfordert, sprenge ihn. Bcgriffliche Kriterien für den rationalen Nachvollzug so feiner Grenzen bietet das Gericht allerdings nicht an.

Das Fehlen rational allgemein vermittelbarer und einleuchtender Kriterien für die Gewichtung der Mehrheits- bzw. Minderheitsinteressen, für das Maß des »unerläßlichen Minimuns« an Zwang, welches der jeweiligen Minderheit zuzumuten ist, wird man in zahlreichen ähnlichen Fällen beobachten - gleichgültig, ob es sich um die Befreiung mohammedanisch-türkischer Schülerinnen vom koedukativen Sportunterricht ${ }^{26}$, um die Durchsetzung des Schächtungsverbotes ${ }^{27}$ oder um die Gewährung von "Kirchenasyl ${ }^{28}$ für abzuschiebende Asylbewerber oder aber um die Mehrfachbestrafung der sogenannten Totalverweigerer handelt.

Dabei fällt den staatlichen Instanzen die Entscheidung auf der Ebene der Verwaltung wie auf der der Justiz relativ am leichtesten dann, wenn die Verwcigerung des Gesetzesgehorsams auf einer singulären, individuell klar bestimnten Gewissensentscheidung beruht, wenn somit wirklich die Ausnabme auftritt, an der die Regel zugleich scheitert und sich bestätigt. Hier gibt der Staat nach, z. B. mit scinem Strafanspruch $^{29}$, und es geht dann nur noch um die zweitrangige Frage, ob und welche Ausgleichslast dem "Gewissenstätcr" auferlegt werden darf.

${ }_{2} 3$ BVerfG EuGRZ 1995, 359, 365 li. Sp. Zum Neutralitatsprinzip vgl. K. Schlaich, Neutralitat als verfassungsrechtliches Prinzip vornehmlıch im Kulturverfassungs- und Staatskirchenreche, 1972.

24 BVerfG EuCRZ 1995, 359, 365, re. Sp., ebenso schon BVerfGE 41, 29, 51 (1975!).

25 Schulgebetsbeschluß vom 16. 10. 1979, BVerfGE \{2, 223 .

26 BVerwGE 94, 82.

2) Zum Problem: T. Kuhl/P. Unruh, Tierschutz und Religıonsfreiheit am Beispiel des Schachtens, DOVV 1991, 94 ff. mit Rechtsprechungsnachweisen.

Ferner F. Hufen, Fundamentalismus als Herausforderung des Verfassungsrechts und der Rechtsphilosophie, in: StWissStPraxis 3 (1992), 45s ff., 463, dort westere Beispiele.

28 Vgl. H.F.Lisken, sKirchenasyle gegen Abschiebung?, POLIZEI-heute 1995, $168 \mathrm{ff}$

29 Vgl. BVeriGE 32, 98 (Gesundbeter). 
Das wahre Ausmaß des Problems, seine verfassungstheoretische Dimension, rückt erst in den Blick, wenn die "Abweichler « nicht (mchr) als singuläre Ausnahmeerscheinungen "verkraftet w werden können, sondern als eine minoritäre Gruppe wahrund ernstgenommen werden müssen. ${ }^{30}$ Eine Verschärfung der ungclösten Fragen ergibt sich, wenn eine Mehrzahl gegeneinander konkurricrender Minderheitengruppen-man denke an PKK-Kurden gegen islamisch-fundamentalistische Türken - das "für alle geltende Geseť" aus unterschiedlichen Motiven in Frage stellen, wcnn, mit anderen Worten, der Multikulturalismus der Gesellschaft die Anomie-Tendenzen zum Dauerproblem werden läßt. Der libcrale Rückıug auf den kleinsten gemcinsamen Nenner allgemein geteilter Wertiiberzeugungen, den das Bundesverfassungsgericht hier noch erfolgreich vorzuführen vermag, reicht dann nicht mehr aus, wenn es darum geht, religiös, ideologisch oder politisch bedingte latente Bürgcrkriegsfronten von den Ursachen her abzubauen.

\section{II.}

Die hier bewußt in ihren negativen Entwicklungsperspektiven gezcichnete Situation führt zu der Frage nach möglichen Alternativen zum bisherigen Verfahren. Dieses kann als eine nachträgliche Ad-hoc-Korrektur des demokratischen Prozcsses durch eine den Grundrechtsstaat zur Geltung bringende Gcrichtsinstanz mit besonderer Machtfülle gekennzeichnct werden. Hier werden verschiedene Denkansätze interessant, die alle in $\mathrm{dem}$ Versuch übcrcinstimmen, den inneren Zusammenhang von Rechtsstaat und Demokratie' neu und chancenreich im Hinblick auf die crforderlichen Problemlösungen zu bestimmen.

Lassen Sie mich an einen Klassiker anknüpfen: Niemand hat in der Frühphase der über drcihundertjährigen Tradition der modernen Staatstheorie den legitimierenden Zusammenhang zwischen individueller Freibcitssicherung - wir würden heute vom Prinzip des materiellen Rechtsstaats sprechen - und der Tcilhabe am Prozeß demokratischer Willensbildung schärfer durchdacht als J.J. Rousseau. Die Qualität eines Staates, unabhängig von seincr monarchischen, aristokratischen oder demokratischen Regierungsform, als Republik ${ }^{32}$ steht und fällt mit der Rüickführung aller seiner Hoheitsakte auf la loi, auf das Gesetz. Dabei dürfen wir "Republik « mit "freiheitlicher Bürgerstaat", näherungsweise sogar mit "Civil society " wiedergeben. Den Bcyriff des Gesetzes kennen wir aus der rule of law, also der Rechtsstaatskonstitution der amcrikanischen Unabhängigkeitsbewegung zur Bcgründung der Gewaltentrennung: "... to end it may be a government of laws, and not of men, «33 also als Absage an jegliche persönliche Willkürherrschaft. So wichtig dieses Moment auch ist, so genügt es doch nicht, dem Gesetz die Qualität als Instrument der Freiheitssicherung und damit seine Legitimität zu verbürgen. Dies leister, so die Theorie des Genfers, das Gesetz nur und insofern, als es im strengen Sinne seinen Begriff erfüllt. Dieser ist durch eine doppelte Allgemeinhett gekennzeichnet. Der Wille der Urheber des Gesetzes muß ebenso "allgemein " ("gesamt $\ltimes$ ) sein wie sein Gegenstand, die

30 Eine differenzıerte Phanomenologic der Minderheiten bictet: A. P'ızzorusso, Minoranze e maggioranze, Torino 1993.

${ }_{31}$ So explizit J. Habermas: Uber den internen Zusammenhang von Rechtsstaat und Demokratie, in U. K. Preuß (Hrsg.), Zum Begriff der Verfassung 1994, S. 83 ff.

32 J.-J. Rousseau, Du contrat social, Buch 11, Kap 6, Fn. 1: Sogar eme Monarche (Regierungsform) kann cine Republık (Volk als Souveran) seın.

33 Verí. von Massachusetts, vom 2.3.1780, sect. XXX. 
Regelungsmaterie. ${ }^{34}$ Nur wenn alle über etwas abstimmen, was inhaltlich alle angeht, verdient das Ergebnis den Namen "Gesetz«. Nur dann bleibt die Freiheit jedes Einzelnen gewahrt.

Die volonté génćrale, deren Ausdruck das Gesctz, ist, wie Art. 6 der Erklärung der Menschen- und Bürgerrechte von $1789^{35}$ fast mit den Worten Rousscaus bekräftigt ${ }^{36}$, kommt also nicht durch Mehrheitsbeschlüsse irgendwelcher Repräsentativorgane zustande, sondern nur durch einen Akt in strenger Identität: "Mais quand tout le peuple statue sur tout le peuple, il ne considère que lui-même «. ${ }^{37}$ Normgeber und Normadressaten müssen identisch sein, nur dann ist auch die Regelungsmaterie universell, nur dann liegt ein Gesctz vor, hinsichtlich dessen sich die Frage erübrigt, ob cs "gerecht « sei oder nicht. ${ }^{\text {s }}$ Über die ticfe Kluft zwischen seinem theoretischen Identitäts-Rigorismus und der historischen Wirklichkcit (wir fügen hinzu: bis auf unsere Tagi') ist Rousscau sich völlig im klaren. Wenn man die Dinge genau betrachtet, meint er, wird man wenige Nationen (Staaten) finden, die "Gesetze" haben. ${ }^{39}$

Die Allgemeinheit des Gesetzes nach Subjekt und Gegenstand als Kriterium seiner Gerechtigkeit und Legitimität begegnet uns wieder bei Immanuel Kant, dem cigentlichen Begründer der deutschen Rechtsstaatstheorie. Gesetzgeber ist für ihn freilich der Monarch, aber der "Probierstein der Rechtmäßigkeit eines jeden öffentlichen Gesetzes ist der gedachte hypothetische Wille des gesamten Volkes..$^{\circ}$ Was bei Rousseau voluntaristisch als "universalité de la volonté konstruiert wird, crschcint bei Kant in vernunftidealistischer Argumentation. Das Gesetz muß für al le gleich gelten, weil alle dem gleichen Prinzip der Sittlichkeit und den Regeln einer für alle gleichen Vernunft unterworfen sind.

Obschon so gut wie nirgendwo im strengen Rousseau'schen Sinne verwirklicht, beherrscht das Allgemeinheitspostulat die Verfassungsthcorie wie die Verfassunggebung bis heute. Das Grundgesetz von 1949 fordert für Grundrechtscinschränkungen ein »allgemein geltendes Gesetz und wiederholt im übrigen die Errungenschaft der Französischen Revolution, daß alle Menschen vor dem Gesetz gleich sind." Sehr zu Recht hat Hasso Hofmann demgegenüber hervorgchoben, daß diese wohlklingende Formel »wirklich sinnvoll « nur sein kann, solange das Gesetz »im wesentliclien noch als Ausdruck einer kraft Sachlogik oder Sittlichkeit allgemeingültigen Regelhaftigkeit angesehen wird «. ${ }^{22}$ Die Gleichheit »vor dem Geserz« als rechtsstaatlich gleiche, willkürfreie Gesetzesanwendung auf alle, die es angeht, beginnt nämlich leerzulau$\mathrm{fen}$ in dem Maße, in dem die Gesetze als solche wungleich ", nicht mehr generell, sondern nur noch "speziell « für immer stärker individualisicrte Sachvcrhalte gemacht werden. Die hiermit angesprochene Problematik der später sogenannten

34 J.-J. Rousseau, Du contrat social, II, 6: „la loı réunissant l'universalıté de la volonté et celle de l'objet .... IV, 2: Die Burger wollen immer die volonté générale (aber ste konnen sich uber ihren Inhalı tauschen)

35 Erklarung der Menschen- und Burgerrechte $1789 / 1791$, Art. 6, S. 1-3: Das Gesetz ist der Ausdruck des allgemeinen Willens. Alle Burger haben das Recht, personlıch oder durch ihre Vertreter an seiner Formung mitzuwrken. Es soll fur alle gleach sein, mag es beschutzen, mag es bestrafen.

36 Du contrat social, III, is: "I.a loi n'étant que la déclaration de la volonté générale ...**

37 Du contrat social, II, 6.

38 Ebenda.

39 Du contrat social, III, is.

40 I. Kant, Uber den Gemeinspruch.... II, Folgerung, Werke Band V1, 1964, S. 153; J. Maus, Zur Aufklarung der Demokrauetheorie, 1992, bes. S. 191 ff., 203 ff.

41 Art. 19 Abs 1 und Art. 3 Abs. 1 GG. S. dagegen z. B. Art, 4 \$. 1 Schwelz. Verf.: Alle Schweizer sind vor dem Gesetz gleich.

42 H. Hofmann, Das Postulat der Allgemeınheit des Gesetzes, in: C. Starck (Hrsg.), Die Allgemeinheit des Gesetzes, Gottingen 1987, S.9ff., 28 . 
"Maßnahmegesetze «"3 hat, längst vor unscrem Zeitalter des Multikulturalismus, Hermann Heller 1927 in scincm berühmten Referat übcr den "Begriff des Gesetzes in der Reichsverfassung " aufgegriffen und konscquent dahin entschieden, "mit der Unmöglichkeit der Durchlegalisierung aller Staatstätigkeit in persönlicher und sachlicher Beziehung " müsse "übrigens auch das Postulat der Allgerneingültigkeit des Gesctzes fallen «.44 Allerdings versucht Heller, die Allgemeinhcit des Gesctzes auf der Seite der Urheberschaft insofern zu "retten «, als er nur dic, "aber auch alle von der Volkslegislative gesetzten obersten Rechtsnormen « als Gesetz anerkennt. is

$I I I$.

Dic immer öfter zu beobachtenden Anomie-Tendenzen, die ich zu Beginn skizziert habe, sind nur zum geringsten Teil Ausdruck der Lust an praktizierten Regelverstoß, vielleicht Ausdruck der gruppengebundenen Identitätssuche in der Negation der widerstandslosen Anpassung an cine als Zwang empfundenc durchregulierte und verwaltete Wclt. ${ }^{+6}$ Für dic Hannoveraner "Chaos-Tage " mag dies zutreffen, und Jugendpsychologen und Sozialarbeiter werden sich ebenso professionell wie oft erfolglos dieses und ähnlicher Phänomene annehmen. Für die religiös, kulturell und cthnisch motivierten, gruppenbezogen auftretenden Verweigcrungen des Gesetzesgehorsams kann die Lösung nur in eincr Neuordnung der Beziehung dieser Bürger zuweilen werden sic als "Mitbürger « abqualifiziert - zum Gesetz liegen. Wir müssen erkennen, daß der hoheitliche Befehl, der ihnen als "Gesetz "gegenübertritt - und der freilich vom Parlament in aller Form rechtens beschlossen worden ist - in ihren Augen, nach ihren religiös-ethisch-kulturellen Maßstäben, weder als volonté générale noch als Ausdruck einer allgemeinen sittlichen Vernunft erscheint, sondern als Oktroi einer ihnen fremden Gewalt. Dic Gesetze, die sie erfahren, sind »universell« weder hinsichtlich der Willen, von denen sie ausgehen, noch hinsichtlich des geregelten Gegenstandes. Sie sind Ausdruck einer "überwältigenden Mehrheit«, nicht ciner sinnvermittelnden Allgemeinheit und nicht einmal der Beginn eines Dialogs.

In dieser scheinbar ausweglosen Situation erscheint es erlaubt, Rat bei einigen Sozialphilosophen zu suchen, die gegenwärtig zu den angeschensten Diagnostikern zählen. Fs ist kein Zufall, daß wir auf die in den USA und in Kanada seit langem geführte Kommunitarismus-Liberalismus-Diskussion rekurrieren (müssen), haben sich doch dort dic Probleme des Multikulturalismus früher und in ganz anderer Schärfe gestellt als hierzulande. Hier wie dort findet die sachlich freilich alte Feststellung Michael. Walzer's Zustimmung, die heutige demokratische Staatsform biete nicht vielen Menschen dic Möglichkeit, sich im Sinne Rousseau's sclbstzubestimmen. "Staatsbürger sind Zuschauer, die wählen ", variiert Walzer einen alten Schumpeter'schen Gedanken.47 Wie steht es dann aber erst, so müssen wir die Frage

43 E. Forsthoff, Uber Maßnahme-Gesetze, in: Gedachtnisschrift fur W. Jellinek, 1955, S. $221 \mathrm{ff}$.

${ }_{44} \mathrm{H}$. Heller, VVDStRL 1928 , Heft $4,9^{8 \mathrm{ff}}, 117$.

45 Heller (Fn. 44), 118. Inweweit das BVerfG mit seiner Rspr. zum Maßnahmegesetz auf dieser Lınie steht, vgl. BVerfGE 25, 371 (lex Rhenstahl), soll hier micht untersucht werden. Jedenfalls wird Art. 19 Abs. I sehr restrikuv interpretiert und ist deshalb praktısch wirkungslos. S. Denninger, AK-GG, Art. 19 I, Rdnr. 6.

46 Andere Teilaspekte des komplexen Gesamtproblems beleuchtet: W. Heitmeyer, Rechtsextremistische Orientuerungen bei Jugendlichen, 4. Aufl. 1992.

47 M. Walzer, Was heißt zıvile Gesellschaft? in: B. van den Brınk/W. van Reijen (Hrsg.), Burgergesellschaft, 
zuspitzen, mit der Selbstbestimmung der vielen Millionen "Zuschauer», die nicht einmal wählen dürfen? Es ist Walzer zu danken, daß er - wie früher schon Hannah Arend $t^{8}$ - die fundamentale Bedcutung des Rechtes, "Mitglied "zu sein, im Staate also dic Staatsbürgerschaft besitzen zu dürfen, immer wieder betont hat. ${ }^{49}$ Unter allen anderen wirklichen und möglichen Mitgliedschaften genießt die Staatsbürgerschaft cinen "gewissen praktischen Vorrang", weil der Staar ein entscheidendes Kampfmittel geworden sei, un das gemeinsame Lcben zu gcstalten. Will man nicht dem permanenten Bürgerkrieg vcrfallen, kann diese Gestaltung nur im Medium des Rechts erfolgen. Jede Art von Mitgestaltung setz.t daher den Status als Rechtsperson, als glcichbcrechtigter "Rcchtsgenosse*, als Mitglied der Rechtsgemeinschaft, als Staatsbürger voraus.

Das Reche und die faktische Möglichkeit der politischen Mitsprache sind die, fast möchtc man sagen: selbstverständlichen Voraussetzungen auch in den Theoriekonzepten jener beiden Autoren, die bei aller gegenseitigen Kritik zu erstaunlichen Parallelen in den Folgerungen gelangen: Ronald Dworkin und Jürgen Habermas. Dworkin geht - wie der Kruzifixbeschluß des Bundesverfassungsgerichts - von dem Spannungsverhältnis z.wischen demokratischem Mehrheitsentscheid und der die Menschenrechte der Minderheit schützenden gerichtlichen Normenkon?rolle aus. Aber in cincr, in Dworkin's Terminologie, "gemeinschaftlichen « und nicht bloß »statistischen « Konzeption von Demokratie erweisen sich scheinbar demokratiebeschränkende Individualrechte in Wahrheit als "demokratieermöglichend «. M. a. W.: Die "gemeinschaftliche Demokratie", welche Minderheiten zu integrieren vermag, setzt die Gewährlcistung des Rechtsstaates voraus. ${ }^{50}$ Umgekebrt hat Jürgen Habermas scine These einmal salopp auf die Formel gebracht: "Kein Rechtsstaat ohne radikale Demokratic. "s' Aber dies ist im Sinne wechselseitiger Bedingtheit zu verstehen: Die Idee der Volkssouveränität und die Idee der Menschenrechte oder: öffentliche und private Autonomie der Bürger verwirklichen sich miteinander und durcheinander. ${ }^{22}$ Die bei beiden Autoren grundlegende Erkenntnis über das, juristisch gesagt, funktionale Synallagma von Demokratieprinzip und McnschenrechtsStatus hilft in unserem Zusammenhang allerdings nicht weiter als zur Überwindung der, in Walzer's Worten, ngebräuchlichsten Form von Despotismus in der menschlichen Geschichte «"3, nämlich der Herrschaft von Staatsbürgern über Nichtstaatsbürger, von Mitgliedern einer Gemeinschaft über in ihr lebende Fremde. Fine ganze Reihe zusätzlicher Voraussetzungen muß erfüllt sein, wenn die Koexistenz religiösethnisch-kulturell verschiedener Gruppen in einer demokratischen Rechtsgemeinschaft nach beiden Richtungen hin gelingen soll: a) in Richtung auf dic Frhaltung einer von der Mehrheit abweichenden Gruppenidentität und b) in Richtung auf die Hervorbringung und Fortbildung einer integricrenden, von allen Gruppen anerkannten Rechtsordnung. Die erste fordert ein Maß wünschbarer Vielfalt, die zweite ein Maß notwendiger Einheit. Weder für das eine noch für das andere läßt sich abstrakt-apriorisch, ohne Rücksicht auf die konkrete historische Situation eine nähere Bestimmung treffen. Vielmehr muß man sehen, daß wir uns mit dieser doppcl-

Reche und Demokratie, 1995, S. 44, 65; J. A. Schumpeter, Kapitalısmus, Sozialısmus und Demokratic, Bern 1946, bes. S. $427 \mathrm{ff}$.

48 H. Arendr, Elemente und Ursprunge totaler Herrschaft, 1962, S. 438 ; s. auch E. Denninger, Menschenrechte und Grundgesetz, 1994, S. 82.

49 Walzer (Fn. 47), S.65; ferner: Spharen der Gerechtigkeit 1992, S. 65 ff., 107.

so R. Dworkin, Gleıchheit, Demokratie und die Verfassung: Wir, das Volk, und die Richter, in: U. K. Preuß (Hrsg.), Zum Begniff der Verfassung, 1994, S. 17, ff.

51 J.Habermas, Die Normalitat einer Berliner Republık, 1995, S. 79.

sz J. Habermas, Uber den internen Zusammenhang von Rechtsstaat und Denokratie, in: Preuß (Hrs:.), Zum Begriff der Verfassung, 1994, S. 83 ff., $90 \mathrm{ff}$.

53 Walzer, Spharen der Gerechugkeit, 1992, S. 107. 
scitigen Frage im Zentrum der Auscinandersetzung zwischen Liberalen und Kommunitaristen bewegen; so daß selbst die vollständige Kenntnis der konkreten Situation nicht zu einem allgemcin akzeptierten Maßstab verhelfen würde. Immer wird es einige geben, die Schutz und Fördcrung der Autonomie und Identität minoritärer Gruppen als vorrangig betrachten, während andere ein höheres Maß von Integration, Assimilation und Anpassung fordern. Viel hängt hier von dem rcligiöskulturellen Selbstbewußtsein der Minderheit und von ihrer Fähigkeit zum Dialog mit der umgebenden Mehrheitskultur ab. Für die vielhundertjährige Identitätsbewahrung der jüdischen Kultur in der Diaspora hat Amos Funkenstein jüngst überzeugend gezeigt, daß die schlichte Entgegensetzung von Assimilation und "Selbstbehauptung oder "Selbstverwirklichung das Phänomen nicht adäquat zu beschreiben vermag, daß vielmehr die beiden polaren Tendenzen Teile eines dialektischen Prozesses sind. ${ }^{.4}$ Und von einer ganz anderen Fragestellung aus, nämlich im Hinblick auf einen für die Reform des amerikanischen Erziehungswesens folgenreichen wertenden Kulturenvergleich kommt Charles Taylor zu einer ähnlichen Feststellung: „Zwischen der unechten homogenisierenden Forderung nach Anerkennung einer grundsätzlichen Gleichwertigkeit einerseits und der Selbsteinmauerung in ethnozentrischen Maßstäben andercrseits muß es noch etwas anderes geben. "ss

Ohne falsche Hoffnung also auf einen "Stein der Weisen ", den es hier nicht geben kann, finden wir bei den sozialphilosophischen Experten einige richtungsweisende Hinweise, die dem mit dem multikulturellen Pluralismus konfrontierten Gesetzgeber von Nurzen sein mögen. Von den drei Prinzipien, nämlich der Partizipation, der gleichen Rücksichtnahme auf die Interessen aller Mitglieder und der Selbständigkeit ihres moralischen Urteils, welche R. Dworkin als Existenzbedingungen der gemeinschaftlichen Demokratie formulicrt ${ }^{56}$, stärken dic bciden let $/ t e r e n$, das Prinzip der Rücksichtnahme und das der Selbständigkeit, die Tendenz zur Wahrung der Gruppenidentität. Demgegenüber wirkt das Prinzip der Partizipation in Richtung auf Integration der Rechtsordnung, nicht nur unmittelbar durch gleiche Teilhabe an der Selektion der normsct\%cnden Repräsentanten, sondern auch mittelbar durch Erzeugung eines Bewußtseins verpflichtender Zugchörigkeit (Dworkin spricht sogar von der "integrierten kollektiven Verantwortung einer Gruppe", welche der Einzelne mitzutragen habe. ${ }^{57}$ )

Einen besonders fruchtbaren, für die Gesetzgebung im Kultur-, Erziehungs- und Bildungsbereich hilfreichen Gedanken bringt J. Habermas in die Diskussion ein, indem er die Entkoppelung der ethischen Integration von Gruppen und Subkulturen von der Ebene der abstrakten, walle Staatsbürger gleichmäßig erfassenden politischen Integration " fordert. ${ }^{88}$ Darnit gibt er einerseits der gruppenbezogenen Identitätsbildung und -wahrung Raum, hält aber andererseits am Universalismus der Rechtsprinzipien fest, dic allerdings ihrerseits in einer gemeinsamen politischen Kultur »sozusagen verfassungspatriotisch eingebetter sein « müssen. Dic Trennung der heiden Integrationsebenen wird in der Rechtspraxis erheblichen Abgrenzungsschwierigkeiten begegnen. Doch kann der Geserzgeber das Konzept als Dirckrive verstehen, in Fragen der Kultur, der Religion, der schulischen Erziehung und überhaupt der Entwicklung ethischer Vorstellungen mit allgemeinen, homogenisierenden

54 A. Funkenstein, The Dialectics of Assimilation, in: Jewish Soctal Studies, I, Nr. 2, 1995, 1 ff. Den Hinveıs verdanke ich Ora Limor, Jerusalem.

5s C. Taylor, Multikulturalismus und die Politik der Anerkennung, 2. Aufl. 1993, S. 70.

s6 Dworkin (Fn. 50), S. 171 ff., 191 ff.

s7 Ebd. S. 197.

s8 Habermas in: Taylor (Fn. 55), S. 177 ff., 179. 
Regelungen Zurückhaltung zu üben. Er soll sich um eine "Politik der Differenz «", der wechselseitigen Anerkennung des jeweils Besondercn, Anderen bemühcn. Eine hegemoniale Mehrheitskultur, welche sich kraft der Majoritätsregel in den staatlichen Institutionen "häuslich cinrichtet «, würde schon die Chance gleichberechtigter Anerkennung der Minderheit(en) zunichte machen.

Gelingt es auf diese Weise, der "Selbstcinmauerung in ethnozentrischen Maßstäben ${ }^{60}$ (oder, im Jargon der US-Debatte: dem Bildungskanon der "toten weißen Männer «, von Platon bis John Dewey) zu entgehen, so gilt es gleichzeitig, der Selbstauslieferung an militante intolerante Fundamentalismen vorzubeugen. Eines der wichtigsten Ergebnisse der in Europa jahrhundertelang bis zur Frschöpfung und bis zur besseren Finsicht getührten Glaubenskriege, eine der unersetzlichen Errungenschaften der Aufklärung ist das Erlernen der Fähigkeit zur Toleranz, d.h. der Fähigkeit, absolute subjektive Glaubensgewißheit mit ihrer Relativiering in der Anerkennung einer anderen, fremden Glaubensgewißheit zu verbinden. ${ }^{61}$ Längst nicht alle religiösen Bewegungen der Erde haben diescs Stadium der Religiosität, exemplarisch von Lessing's Nathan verkörpert, schon erreicht. Es zeugt von Stärke, nicht von Sch wäche der eigenen Glaubensgewißheit, wenn man den Glaubensfremden voll akzeptieren kann. Aber diese Haltung kann situativ zu einer Hilf-oder Wehrlosigkeit gegenüber militanten Aktionen fundamentalistischer Fanatiker führen, die nicht bereit sind, die Spiejregcln demokratischer Verfahren einzuhalten. Das für alle geltende allgemeine Gesetz schützt in seiner rechtsstaatlichen Funktion auch die Fundamentalisten, die irgendeinen elitären Gottes- oder Einparteienstaat anstelle der pluralistischen Demokratie errichten wollen. Voraussetzung ist, daß sie das inncrstaatliche Gewaltverbot, die Strafgesetze und die Rechtsordnung im allgemeinen respektieren. Die liberalc rechtsstaatliche Demokratie begibt sich insoweit bewußt in eine nicht ungefährliche Asymmetrie: Sie leistet mchr, als sic von den Begünstigten im Gegenzug zu erwarten hat. Denn dicse vergelten Rechtssicherheit mit Illoyalität, im Extremfall Toleranz mit Gewalt. Man kann die Probe aufs Exempel machen, indem man dic Frage stellt, ob eine gedeihliche pluralistische Demokratie aus lauter Mitgliedern denkbar ist, die vor-aufklärerisch-intolerant, eben "fundamentalistisch « eingestellt sind. ${ }^{62}$ Die Antwort lautet: Nein. Diese Bürger wären unfähig, einander in ihrer Andersheit anzuerkennen, unfähig, einander solidarisch zu begegnen. ${ }^{63}$ Blutiger Bürgerkrieg wäre unausweichlich.

Um einen (Rechts-)Staat hervorzubringen, meinte Kant, genügte auch ein Volk von Teufeln, wenn sie nur Verstand haben. ${ }^{64}$ Für eine multikulturelle, gemeinschaftliche Demokratie reicht das nicht aus. Es genügt nicht, eine beliebige Menge von Menschen auf einem begrenzten Territorium mit formal gleichem Gesetzgebungsrccht zusammenzubringen, um eine demokratische Rechtsgemeinschaft zu erzeugen. Schon A. de Tocqueville hatte, am Beispiel Amerikas, entdeckt, daß eine individualistische egalitäre Demokratie der Basis in zahllosen kleinen und kleinsten Vereinigungen bedarf und daß es gilt, die "Kunst der Vergesellschaftung ", "l'art de s'associer" zu üben und zu entwickeln, soll die Demokratie überleben. ${ }^{65}$ Ohne den großen Franzosen zu zitieren, spricht $M$. Walzer heute von der "Kunst des Verbindens" als

59 Taylor (Fn. 55), S. 29; Walzer, Zivile Gesellschaft und amerikanusche Demokratıe, 1992, S. 228 ff.

60 Taylor (Fn. 55 ), S. 70 .

61 Vgl. H. Kelsen, Vom Wesen und Wert der Demokratue, 2. Aufl. Tubingen 1929.

62 F. Hufen (Fn. 27), 45 sff., geht her sehr wert.

$6_{3} \mathrm{Zu}$ Begriff und Funktion von "Solidantat * vgl. E. Dennunger, Verfassungsreche und Solidaritat, KritV 1995,7 ff.

64 I. Kant, Zum ewigen Frieden, II. Abschnit,, 1. Zusatz, Werke, Band VI, Darmstadt 1964, S. 224

65 A. de Tocqueville, De la Démocratıe en Amérıque, 2 Band, 2. Teıl, Kap. 5 am Ende, 13. Aufl. Parıs 1850, S. 124 f. 
einer entscheidenden Voraussetzung der Demokratie. ${ }^{66}$ Sein scritical associationalism " geht von einer Wechselwirkung zwischen demokratischem Staat und ziviler Gesellschaft aus. Diese "Civil Society", aus der "Kunst des Sichvereinigens " erwachsen, ist unsere Chance, unsere ständige Aufgabe. Aber das ist ein anderes sweites Fold.

66 Walzer, in: van den Brink/van Rejen (Fn. 47), S. 44 f?., 55. 